(including Attention Deficit Hyperactivity Disorder -ADHD), but no FASD diagnosis. FASD is associated with a large number of comorbid conditions, including an 80-fold increased risk of sensorineural hearing loss. Using a contemporary UK birth cohort we examined the association between maternal alcohol consumption in pregnancy, hearing problems at age 3 and later ADHD.

Methods Analysis of the UK Millennium Cohort Study (MCS), based on 9179 children participating in 3 survey sweeps (9 months, 3, 7 years old). The main outcome was parentalreported hearing problems, at age 3 . We also examined selfreported diagnosis of $\mathrm{ADHD}$ at age 7 . Incident rate ratios (IRRs; 95\% confidence intervals [CI]) were estimated using Poisson regression, according to maternal drinking in pregnancy in units per week (categorised as none, 1-7, 8-14 and 15 or more) adjusted for measures of childhood socioeconomic conditions. Analyses were conducted using Stata/SE with svy commands to account for the sampling design and attrition.

Results At age 3, 1328 children (14.4\%) had reported a hearing problem with the most common of these being otitis media and unspecified hearing loss. Children of mothers who reported drinking 15 or more units per week of alcohol in pregnancy were twice as likely to have hearing problems compared to mothers who reported never drinking in pregnancy (aIRR1.9(95\% CI[1.07, 3.39])). Risk of ADHD diagnosis at age 7 was also elevated in children of mothers who drank 15 or more units per week though this was not statistically significant at the 5\% level (aIRR2.79(95\% CI[0.91, 8.57])). Children with hearing problems at age 3 were 3.5 times more likely to have a diagnosis of ADHD at age 7 (aIRR3.49 (95\% CI[1.92, 6.32])).

Conclusion In a representative UK child cohort we found a significant prevalence of parental reported hearing problems at age 3 which was associated with high maternal alcohol consumption in pregnancy. Hearing problems were associated with a higher risk of ADHD. Self-reported data of both alcohol consumption and hearing problems is a limitation of this study. Maternal alcohol consumption is rarely captured in child health records in the UK making follow-up of potentially exposed children difficult. Further investigation in children with hearing loss could lead to earlier diagnosis of neurodevelopmental conditions, including FASD, and provide opportunities to intervene and improve outcomes.

\section{OP55 THE IMPACT OF NEWBORN SCREENING ON OUTCOMES AND INEQUALITIES IN CYSTIC FIBROSIS: A UK REGISTRY BASED STUDY}

${ }^{1}$ DK Schlüter*, ${ }^{2} \mathrm{C}$ Dryden, ${ }^{3} \mathrm{KW}$ Southern, ${ }^{1} \mathrm{PJ}$ Diggle, ${ }^{4} \mathrm{D}$ Taylor-Robinson. ${ }^{1} \mathrm{CHICAS}$, Lancaster Medical School, Lancaster University, Lancaster, UK; ${ }^{2}$ Department of Paediatrics, University Hospital Wishaw, Wishaw, UK; ${ }^{3}$ Department of Women's and Children's Health, University of Liverpool, Liverpool, UK; ${ }^{4}$ Department of Public Health and Policy, University of Liverpool, Liverpool, UK

10.1136/jech-2018-SSMabstracts.55

Background Cystic fibrosis (CF) is the commonest inherited disease in white populations, and as a classically inherited genetic disease, there is no socio-economic gradient in incidence. Despite this, we have previously demonstrated early inequalities in CF outcomes such as reduced growth and poorer lung function in children from more socio-economically disadvantaged areas. In the present study, we used data from the UK CF Registry to examine the impact of newborn bloodspot screening (NBS), introduced in 2007, on inequalities in clinical outcomes in children with CF born in the new millennium.

Methods We carried out longitudinal analyses of data on 4117 individuals with CF born between 2000 and 2014 who are captured in the UK CF Registry. Clinical outcomes were the trajectories of lung function measured using percent predicted $\mathrm{FEV}_{1}\left(\mathrm{ppFEV}_{1}\right)$ from age five, weight and body mass index (BMI) z-scores from age one, and time to chronic Pseudomonas Aeruginosa (PA) infection. We developed longitudinal models for $\mathrm{ppFEV}_{1}$, weight, and BMI and a time-to-event model for PA infection to assess the association of NBS with outcomes and potential interactions with childhood socio-economic conditions (SECs), measured by the index of multiple deprivation, whilst adjusting for sex, genotype, birth cohort, ethnicity, and pancreatic insufficiency.

Results Complete data for the analyses of the effect on lung function, weight, BMI and time to chronic PA infection were available for 2267, 3424, 3410 and 3428 individuals, respectively. About one third of the individuals were diagnosed by NBS. NBS was associated with a shallower rate of lung function decline $(0.45 ; 95 \%$ CI 0.13 to 0.76 per year), and higher average weight trajectory intercept $(0.14$; $95 \%$ CI 0.06 to 0.23 standard deviations) as well as increased time to chronic PA infection. We found no significant association of NBS with the intercept for lung function or BMI; or with longitudinal trajectories of weight and BMI. There was no significant interaction between NBS and childhood SECs.

Conclusion Analyses of data from a large national CF Registry show that NBS is associated with better lung function and increased weight for all children with $\mathrm{CF}$, but there is no evidence that it has narrowed health inequalities.

\section{Smoking and youth}

\section{OP56 ABSTRACT WITHDRAWN}

\section{OP57 \#DOES PARENTAL SMOKING EXPLAIN IMPACTS OF SMOKE-FREE PUBLIC PLACES LEGISLATION ON YOUTH SMOKING INITIATION IN THE UK?}

PE Anyanwu*, P Craig, SV Katikireddi, MJ Green. MRC/CSO Social and Public Health Sciences Unit, University of Glasgow, Glasgow, UK

\subsection{6/jech-2018-SSMabstracts.56}

Background Evidence on the impact of the smoke-free public places legislation in the UK on youth smoking initiation is not well established. Changes in parental smoking behaviour may be a major mechanism by which smoke-free legislation impacts on youth smoking. Smoke-free legislation could also displace parental smoking behaviour into the home (by restricting alternative smoking spaces) or out of the home (by increasing awareness of risks to others from second-hand smoke exposure), potentially either strengthening or weakening its influence. We investigated how much of any impact of the legislation on youth smoking initiation could be explained by parental smoking, and whether associations between parental smoking and youth smoking initiation differed before or after the legislation. 
Methods Longitudinal data from the annual British Household Panel Survey and Understanding Society (1994-2016) were examined with discrete-time event history analyses. 14992 youths contributed data for up to five observations (67 556 person-years) representing ages 11-15 years, with data rightcensored at the year of smoking initiation (or age 15). We examined associations between smoking ban implementation (2006 for Scotland, 2007 for other UK countries) and youth smoking initiation (i.e. trying smoking for the first time), before and after adjustment for parental smoking, and tested for interactions between parental smoking and the ban implementation. Multiple imputation was used to handle missing data. Analyses were adjusted for youth sex, age, UK country, socioeconomic status, and temporal trends in initiation.

Results Youth initiation of smoking was less likely after the implementation of the smoke-free legislation than before (OR: 0.16 ; $95 \%$ CI 0.14 to 0.18 ), and this difference further increased with each year after implementation (OR per year after the ban:0.88; 95\% CI 0.82 to 0.94). Adjustment for parental smoking only marginally attenuated the association with ban implementation (OR: 0.20 ; 95\% CI 0.16 to 0.24 ) and the per-year decrease after the ban (OR: 0.86 ; $95 \% \mathrm{CI}$ 0.80 to 0.92 ) was similar. There was an interaction between the ban implementation and parental smoking ( $p$-value: 0.001) such that parental smoking was more strongly associated with youth smoking initiation before the ban (OR: 1.41 ; 95\% CI 1.26 to 1.58 ) than after (OR: $0.92 ; 95 \%$ CI 0.65 to 1.32 ).

Discussion Changes in parental smoking behaviour did not seem to be a major explanation for the strong impacts of smoke-free legislation on youth smoking initiation, suggesting other mechanisms may be at work. The influence of parental smoking on youth initiation weakened after the ban which may indicate the displacement of parental smoking behaviour out of the home.

\section{OP58 ASSOCIATIONS BETWEEN PARENTAL VAPING AND YOUTH SMOKING AND VAPING IN THE UK: CROSS- SECTIONAL SURVEY FINDINGS}

${ }^{1} \mathrm{MJ}$ Green*, ${ }^{1} \mathrm{~L}$ Gray, ${ }^{1} \mathrm{H}$ Sweeting, ${ }^{2} \mathrm{M}$ Benzeval. ${ }^{1} \mathrm{MRC/CSO}$ Social and Public Health Sciences Unit, University of Glasgow, Glasgow, UK; ${ }^{2}$ Institute for Social and Economic Research, University of Essex, Colchester, UK

\subsection{6/jech-2018-SSMabstracts.57}

Background E-cigarette use has been rising steadily in the UK since 2011. Mounting evidence suggests e-cigarette use ('vaping') is safer than traditional cigarettes ('smoking'), and can aid smoking cessation. Nevertheless concerns are still raised that vaping may re-normalise smoking behaviour and help introduce youth to traditional cigarettes. Parental smoking is long-established as a risk factor for youth smoking, so parental vaping may be an important component of any normalisation effects of e-cigarettes on youth. We estimated associations of parental vaping with youth smoking and vaping.

Methods Data were from the 7th wave of the Understanding Society study, a representative annual panel study of UK households. Youth and parents self-reported current vaping and current and past smoking (parental vaping/smoking coded for any use in couples). Logistic regression analyses were conducted in STATA on 2373 youth aged 10-15 with valid data
(65\% of 3635 interviewed; 51\% female). Models were weighted for study non-response and adjusted for potential confounders (gender, age, UK country, ethnic minority, single parent status, parental education, occupation, income and parental smoking). Where there were interactions between parental vaping and parental smoking (never, ex or current), we estimated ORs within strata of parental smoking. With responses from previous surveys we additionally examined current smoking in youth who had never smoked before $(n=2,243$; i.e. initiation of smoking).

Results Vaping and smoking were rare among youth $(3.5 \%$ and $2.3 \%$ respectively) but more youth had parents who vaped $(11.6 \%)$ or were ex $(39.7 \%)$ or current $(24.9 \%)$ smokers. Parental vaping was not clearly associated with youth vaping after adjustment for confounders (OR: 1.86; 95\% CI 0.70 to 4.96). However, associations between parent and youth vaping varied by parental smoking ( $p$-value for interaction: 0.043 ) being strongest among youth whose parents were exsmokers (OR: 4.99; 95\% CI 0.94 to 26.53). After adjustment, parental vaping was not clearly associated with youth current smoking either (OR: 1.26 ; 95\% CI 0.38 to 4.19), which held across strata of parental smoking (interaction p-value: 0.102). However, parental vaping was associated with youth smoking initiation, even after adjustment (OR: 4.63; 95\% CI 1.29 to 16.58 ), and this too did not vary by parental smoking status (interaction p-value: 0.115 ).

Conclusion There was not strong evidence that parental vaping encouraged youth smoking or vaping overall. However, some indications of increased risk (e.g. for smoking initiation, or among youth whose parents were ex-smokers) warrant further study and monitoring to further inform regulation, especially as increasing numbers of smokers (including parents) switch to e-cigarettes.

\section{OP59 \#HAVE E-CIGARETTES RE-NORMALIZED OR DISPLACED YOUTH SMOKING?: A SEGMENTED REGRESSION ANALYSIS OF REPEATED CROSS SECTIONAL SURVEY DATA IN ENGLAND, SCOTLAND AND WALES}

${ }^{1}$ B Hallingberg*, ${ }^{2} \mathrm{O}$ Maynard, ${ }^{3} \mathrm{~L}$ Gray, ${ }^{4} \mathrm{~A}$ MacKintosh, ${ }^{1} \mathrm{E}$ Lowthian, ${ }^{1} \mathrm{G}$ Moore. ${ }^{1}$ School of Social Science, Cardiff University, Cardiff, UK; ${ }^{2}$ School of Experimental Psychology, University of Bristol, Bristol, UK; ${ }^{3} \mathrm{MRC} / \mathrm{CSO}$ Social and Public Health Sciences Unit, University of Glasgow, Glasgow, UK; ${ }^{4}$ Institute for Social Marketing, University of Stirling, Stirling, UK

\subsection{6/jech-2018-SSMabstracts.58}

Background Small yet significant impacts of e-cigarettes on population smoking cessations rates indicate promise for harm reduction. However, non-smoking young people are increasingly experimenting with e-cigarettes, and while regular use remains rare, arguments for regulation have been driven by fears that e-cigarettes re-normalize smoking. Others counter that e-cigarettes may displace youth smoking and further denormalize it. This study tests whether the secular decline in youth smoking prevalence, as well as perceived smoking norms, slowed or accelerated during the period from 20112015 (when e-cigarettes were emerging but prior to recent moves to regulate their use).

Methods Data were taken from the Smoking Drinking and Drug Use survey in England, Health Behaviour in School Aged Children/School Health Research Network surveys in 